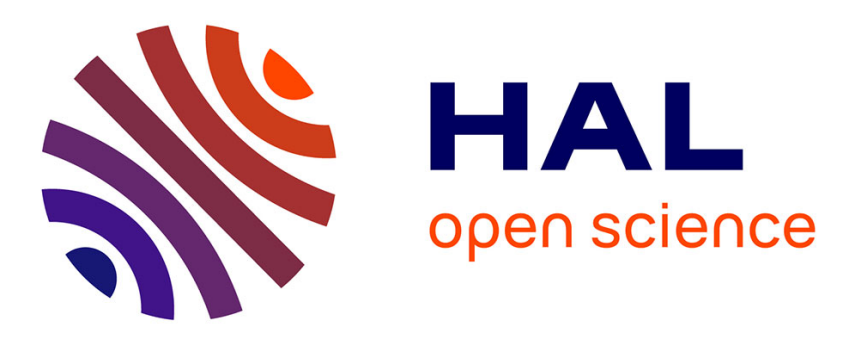

\title{
Terahertz Radiation from Magnetic Excitations in Diluted Magnetic Semiconductors
}

\author{
R. Rungsawang, Florent Perez, D. Oustinov, J. Gómez, V. Kolkovsky, G. \\ Karczewski, T. Wojtowicz, J. Madéo, N. Jukam, S. Dhillon, et al.
}

\section{- To cite this version:}

R. Rungsawang, Florent Perez, D. Oustinov, J. Gómez, V. Kolkovsky, et al.. Terahertz Radiation from Magnetic Excitations in Diluted Magnetic Semiconductors. Physical Review Letters, 2013, 110 (17), pp.177203. 10.1103/PhysRevLett.110.177203 . hal-00975401

\section{HAL Id: hal-00975401 \\ https://hal.science/hal-00975401}

Submitted on 1 Jun 2016

HAL is a multi-disciplinary open access archive for the deposit and dissemination of scientific research documents, whether they are published or not. The documents may come from teaching and research institutions in France or abroad, or from public or private research centers.
L'archive ouverte pluridisciplinaire HAL, est destinée au dépôt et à la diffusion de documents scientifiques de niveau recherche, publiés ou non, émanant des établissements d'enseignement et de recherche français ou étrangers, des laboratoires publics ou privés. 


\title{
Terahertz radiation from magnetic excitations in diluted magnetic semiconductors
}

\author{
R. Rungsawang, ${ }^{1}$ F. Perez,${ }^{2, *}$ D. Oustinov,${ }^{1}$ J. Gómez,${ }^{2, \dagger}$ V. Kolkovsky, ${ }^{3}$ G. \\ Karczewski ${ }^{3}$ T. Wojtowicz, ${ }^{3}$ J. Madéo, ${ }^{1}$ N. Jukam,${ }^{1}$ S. Dhillon, ${ }^{1}$ and J. Tignon ${ }^{1}$ \\ ${ }^{1}$ Laboratoire Pierre Aigrain, Ecole Normale Supérieure, \\ CNRS (UMR 8551), Université P. et M. Curie, \\ Université D. Diderot, 75231 Paris Cedex 05, France. \\ ${ }^{2}$ Institut des Nanosciences de Paris, CNRS (UMR7588), Université Paris VI, Paris 75005, France \\ ${ }^{3}$ Institute of Physics, Polish Academy of Sciences, 02-668 Warsaw, Poland
}

(Dated: February 28, 2013)

\begin{abstract}
We probed in the time domain, the $\mathrm{THz}$ electromagnetic radiation originating from spins in CdMnTe diluted magnetic semiconductors quantum wells containing high mobility electron gas. Taking advantage of the efficient Raman generation process, the spin precession was induced by low power near-infrared pulses. We provide a full theoretical first-principles description of spin-waves generation, spin precession and of emission of the $\mathrm{THz}$ radiation. Our results open new perspectives for improved control of the direct coupling between spins and electromagnetic field, e.g. by using semiconductor technology to insert the $\mathrm{THz}$ sources in cavities or pillars.
\end{abstract}

PACS numbers: 07.57.Hm,75.78.Jp,73.21.Fg,75.50.Pp,78.30.Fs, 78.47.J-,85.75.-d

Spin-based sources of $\mathrm{THz}$ electromagnetic radiation have recently attracted a great deal of attention, especially since spin waves were proposed to provide radiating sources to transmit or modify logical spin-based information[1]. In this context, direct light emission or absorption from the spin degrees of freedom are at the frontier of novel physics[2]. Until now, however, optically $[3-5]$ or magnetically [6] excited spin waves have been primarily investigated in antiferromagnets such as $\mathrm{NiO}$. It is less obvious to expect similar $\mathrm{THz}$ emission from spin excitations in semiconductors, since typical semiconductors are paramagnetic and are limited by much lower concentrations of available spins (by a factor of $10^{-4}$ ), which results in much weaker emitted fields. Even worse, conventional semiconductors are lacking an optical spin mode with a frequency in the $\mathrm{THz}$ range. On the other hand, however, semiconductors - and particularly the engineering of nanostructures with high materials quality - offer an excellent laboratory for controlling and studying the coupling of photon modes with the spin degrees of freedom, and thus for making advances in the spin-based $\mathrm{THz}$ emission.

Here we provide first steps in the experimental and theoretical exploration of spin-based $\mathrm{THz}$ emission from diluted magnetic semiconductors (DMSs) [7, 8] quantum wells. In DMSs, the number of available spins is controlled by the concentration $x$ of magnetic ions (e.g. Mn) incorporated into the nonmagnetic host, so as to form crystals as $\mathrm{Ga}_{1-x} \mathrm{Mn}_{x} \mathrm{As}$ or $\mathrm{Cd}_{1-x} \mathrm{Mn}_{x} \mathrm{Te}$. Moreover, spin excitations occur in the form of mixed modes, in which spins of the free band carriers and those of localized magnetic ions oscillate coherently, in phase (acoustic modes) or out of phase (optical modes) $[9,10]$. The precession frequency of these optical modes, typically follows the spin-split (Zeeman) energy of the carriers, $Z$, which is enhanced by the large exchange interaction with the $d$-shell electrons of the localized Mn ions. Thus the precession frequency can be adjusted in the $\mathrm{THz}$ range both by $x$ and by the DMS exchange integral $N_{0} \beta$ (for holes) or $N_{0} \alpha$ (for electrons).

$\mathrm{THz}$ transient emission from $\mathrm{Ga}_{1-x} \mathrm{Mn}_{x} \mathrm{As}$ in the ferromagnetic state has already been detected by the time domain measurements[11]. However, the spin origin of the radiation was not demonstrated, as the frequency range of the observed transient did not match neither the acoustic nor optical modes frequencies. We preferentially used a well controlled test-bed DMS system made of a two dimensional electron gas (2DEG) embedded in a $\mathrm{Cd}_{1-x} \mathrm{Mn}_{x}$ Te quantum well (QW), which has been introduced [12-15] as an alternative for magnetic excitations study in DMS. In this paramagnetic system the acoustic and optical spin wave modes have been evidenced by Raman spectroscopy [12-15] and time-domain optical pump-probe experiments[16, 17]. Upon application of an external magnetic field $B \mathbf{z}$ parallel to the quantum well plane (see inset of Fig.1 and Ref.[18]), each paramagnetic $\mathrm{Mn}$ atom supports a thermal average spin $\left\langle I_{z}(B, T)\right\rangle$. The spin-split Zeeman energy $Z$ of the QW conduction band, writes:

$$
Z(B, T)=\bar{x} N_{0} \alpha\left\langle I_{z}(B, T)\right\rangle-\left|g_{e}\right| \mu_{B} B,
$$

In Eq.(1), $N_{0} \alpha=-0.22 \mathrm{eV}$ [19], $\bar{x}$ is the effective $\mathrm{Mn}$ concentration (for low $x, \bar{x} \simeq x$ ), and $g_{e}=-1.64$ is the electron Landé factor in CdMnTe $\left(\mu_{B}>0\right)$.

In our experiment, the spin-polarized 2DEGs reside in each of 20 QWs within a $\mathrm{Cd}_{1-x} \mathrm{Mn}_{x}$ Te multi quantumwell structure[18]. The sample exhibit well resolved quantum Hall plateaus and Shubnikov de Haas oscillations. 2DEG sheet densities $n_{2 D}$ and mobilities were found to be $5 \times 10^{11} \mathrm{~cm}^{-2}$ and $2.4 \times 10^{4} \mathrm{~cm}^{2} / \mathrm{Vs}$. The Mn concentration, as determined from Raman measurements[14], is $x=1.75 \%$, which implies that $Z$ 
can be as high as $6.7 \mathrm{meV}(\simeq 1.6 \mathrm{THz})$ for $B=8 \mathrm{~T}$. In this case the 2DEG becomes highly spin polarized with a spin polarization degree $\zeta=\left(n_{\uparrow}-n_{\downarrow}\right) /\left(n_{\uparrow}+n_{\downarrow}\right) \simeq$ $-Z / 2 E_{F} \simeq 40 \%\left(E_{F}\right.$ is the Fermi energy). A circularly polarized near-infrared femtosecond pulse propagating perpendicularly to the quantum well plane (see inset of Fig.1 and Ref.[18]) exchanges angular momentum with the electron spins and the Mn spins through a Raman generation process thanks to strong spin-orbit interaction in the valence states[20, 21]. A collective spin precession starts and within the relaxation time, the spin oscillation radiates an electromagnetic field which is probed by electro-optic sampling. In Fig.1(a), we have plotted for various static magnetic fields, the transient electric field radiated from the sample excited by an optical pulse centered at $763 \mathrm{~nm}$. The electro-optic sampling detection was set to be sensitive to the electric field component parallel to the static magnetic field axis (z axis). A transient, well resolved, oscillation of the emitted field is observed. The amplitude of the electric field oscillation saturates with the external magnetic field as expected (see Fig.1(b) and text below). The emission was measured with an energy per pulse $E_{P} \simeq 70 \mathrm{~nJ} / \mathrm{cm}^{2}$, which is orders of magnitude smaller than that used for $\mathrm{Ga}_{1-x} \mathrm{Mn}_{x}$ As in Ref.[11] $\left(400 \mu \mathrm{J} / \mathrm{cm}^{2}\right)$ or for $\mathrm{NiO}$ in Ref. $[22]\left(20 \mathrm{~mJ} / \mathrm{cm}^{2}\right)$. The power requirements in this work are strongly reduced by the strong spin-orbit interaction occurring in valence states and the sharpness of the optical resonance involved in the Raman generation mechanism. The latter is assured by the recent progress of MBE growth of II-VI semiconductor heterostructures[23].

Illustration of the resonance is given in Fig.2(a), where, for a fixed magnetic field, we tuned the central wavelength $\lambda_{0}$ of the optical pulse across the lowest 2DEG's optical resonance positioned at $763 \mathrm{~nm}$ (as determined by cw optical measurements). The amplitude of the radiated oscillation is maximum at $763 \mathrm{~nm}$. This is a first evidence that the emission originates from 2DEG electrons and not from the CdTe buffer or from the GaAs substrate.

The spin origin of the radiation is inferred from Fig.2(c) where the Fourier-transform of the detected field transient (shown in Fig.1(a)) is plotted for circularly and linearly polarized optical pulses in the presence or absence of the magnetic field. As clearly seen the radiation is detected only when the magnetic field is switched on and when the optical pulse is circularly polarized. This reflects perfectly the symmetry of the inverse Faraday effect [25-27] in a cubic crystal: a circularly polarized pulse exchange a transient magnetic moment along the propagation direction while a linearly polarized pulse has no magnetic effect, but couples to charge excitations only[20]. In addition, in absence of external magnetic field, the out of phase spin wave disappears. These observations also demonstrate that the detected radiation

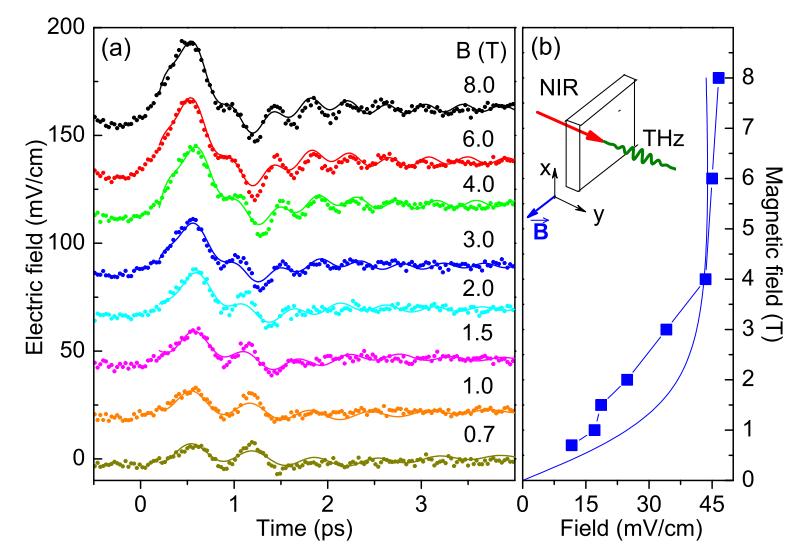

FIG. 1. (Color online). (a) Time-dependent radiated fields for various magnetic fields $(B)$. A vertical offset is added for clarity. Fitted sinusoids are superposed. (b) (squares) Amplitude of the transient oscillation extracted from (a), as a function of the magnetic field. (line) Radiated amplitude calculated for one quantum well (see text). (Inset) Sketch of the experiment: a near-infrared (NIR) pulse (100 fs) with circular polarization is focused, with normal incidence, on the QW structure. The sample is immersed in a bath of superfluid helium inside a split-coil magnet with a static field $B$ up to $8 \mathrm{~T}$ applied along the quantum well plane (parallel to the $z$ direction). The $\mathrm{THz}$ transient electric field is collected along the $z$ direction[18].

cannot be related to a magnetic field enhancement of a radiation originating from charge excitations as described in Ref.[24]. Indeed, the pulse, normally incident and focused on $1 \mathrm{~mm}$ diameter spot, can induce a charge motion only perpendicular to the quantum well plane and the Lorentz force is unable to induce a dipole along the magnetic field axis ( $\mathbf{z}$ axis), which is the detected radiation polarization.

We confirm the spin origin by providing a quantitative treatment of the amplitude of the radiation and the frequencies present in the generated $\mathrm{THz}$ transient, and demonstrate that it supports the data. The impulsive optical electromagnetic field, circularly polarized in the plane $(\mathbf{x}, \mathbf{z})$ of the sample couples to the crystal through the Hamiltonian: $\hat{H}(t)=-e \mathbf{A}(t) \cdot \hat{\mathbf{p}} / m^{*}=\int \tilde{H}_{\omega} e^{i \omega t} d \omega$, where $\mathbf{A}(t)$ is the time dependent vector potential. Before the pulse excites the sample, the crystal is in its equilibrium state $|i\rangle$ where electron and $\mathrm{Mn}$ spins are anti-parallel to the magnetic field. The macroscopic electron spin $\hat{\mathbf{S}}=\sum_{j} \hat{\mathbf{s}}_{j}$ is then: $\left\langle i\left|\hat{S}_{z}\right| i\right\rangle=S_{z i}=\frac{1}{2} n_{2 D} L^{2} \zeta$, where $L^{2}$ is the illuminated QW surface. The macroscopic $\mathrm{Mn}$ spin $\hat{\mathbf{M}}=\sum_{j} \hat{\mathbf{I}}_{j}$, has the equilibrium value $\left\langle i\left|\hat{M}_{z}\right| i\right\rangle=M_{z i}=x N_{0}\left\langle I_{z}(B, T)\right\rangle w L^{2}$, where $w$ is the QW thickness. After its action, through the Raman process, the pulse leaves the system in the state [18, 21]:

$$
|\Psi(t)\rangle=c_{i} e^{-i \omega_{i} t}|i\rangle+\sum_{m} c_{m} e^{-i \omega_{m} t}|m\rangle
$$




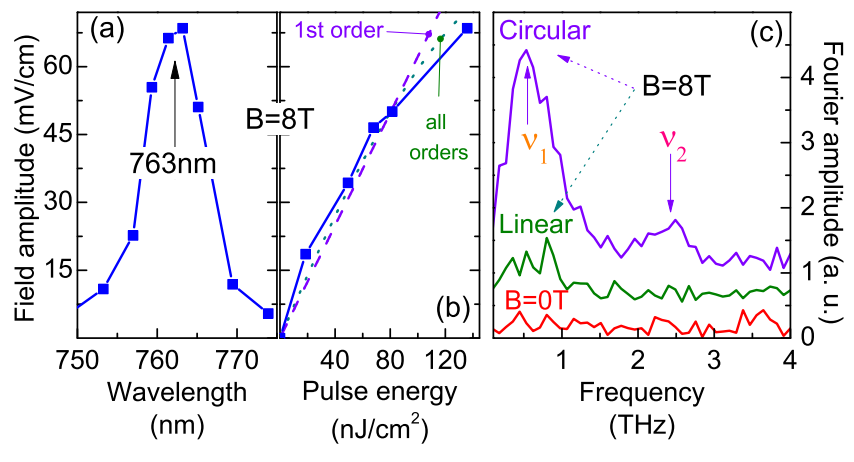

FIG. 2. (Color online). (a) Transient electric field amplitude as a function of central wavelength of the pulse. The pulse energy was $136 \mathrm{~nJ} / \mathrm{cm}^{2}$. (b) Variation of the detected radiated amplitude (squares) with the pulse energy and comparison with the calculations carried to 1st-order with $\operatorname{Eqs}(3)-(4)$ and to all orders[18]. (c) Fourier amplitudes of the electric field when excited by circularly (top line) and linearly (middle line) polarized pulse at $\mathrm{B}=8 \mathrm{~T}$. Two peaks are seen at $0.5 \mathrm{THz}\left(\nu_{1}\right)$ and $2.4 \mathrm{THz}\left(\nu_{2}\right)$ when the pulse is circularly polarized. The bottom line is the result of circular excitation at $\mathrm{B}=0 \mathrm{~T}$. All data in (c) were taken with $82 \mathrm{~nJ} / \mathrm{cm}^{2}$ energy pulses.

where $|m\rangle$ are excited quantum states with eigen pulsation $\omega_{m}$ and $c_{m}$ writes (to lowest order in the field [18]):

$$
c_{m}=i \frac{\pi}{\hbar^{2}} \int \sum_{n} \frac{\left\langle m\left|\tilde{H}_{\omega-\omega_{m i}}\right| n\right\rangle\left\langle n\left|\tilde{H}_{\omega}^{*}\right| i\right\rangle}{\omega_{n i}-\omega+i \eta_{n}} d \omega
$$

In Eq.(3), $|n\rangle$ are intermediate states, $\eta_{n}$ is their decay rate and $\omega_{m i}=\omega_{m}-\omega_{i}$. Note that the optical pulse resonates with the crystal transition when $\omega \simeq \omega_{n}-\omega_{i}$ over a range given typically by $\eta_{n}$. A coherence between $|i\rangle$ and a spin-wave state $|f\rangle$ will be driven, if the pulse spectrum contains two photons separated by $\omega_{f i}$, and if these two photons can together transfer magnetic momentum. For the latter, the spin-mixture in the hole state involved in the interband electron-hole pair intermediate state $|n\rangle$ is essential[18, 20,21]. The cubic symmetry of the CdTe crystal makes the Raman process obeys the general symmetry of the inverse Faraday effect $[26,27]$. It results[18] that $c_{f}=i \int \chi_{f}(\omega)\left\langle f\left|\hat{\mathbf{S}} \cdot \mathbf{A}_{\omega-\omega_{f i}} \times \mathbf{A}_{\omega}^{*}\right| i\right\rangle d \omega$, where $\chi_{f}(\omega)$ is a scalar. Therefore, the circularly polarized pulse couples to the spin degrees of freedom as an effective magnetic field proportional to $\mathbf{A}_{\omega-\omega_{f i}} \times \mathbf{A}_{\omega}^{*}$, which is, vanishing for a linearly polarized pulse, but directed along the propagation direction $\mathbf{y}$ for a circular pulse. Thus, our circular pulse induces an out of equilibrium magnetization and leaves the system in a state such that the average $\mathrm{Mn}$ and electron spins are respectively $\mathbf{M}(t=0)=\left(0, M_{y 0}, M_{z 0}\right)$ and $\mathbf{S}(t=0)=\left(0, S_{y 0}, S_{z 0}\right)$ $(\mathbf{M}(t)=\langle\Psi(t)|\hat{\mathbf{M}}| \Psi(t)\rangle)$. Both macroscopic spins will further experience a spontaneous precession with a relaxation towards their equilibrium states.

In a semi-classical picture, the spin magnetization is

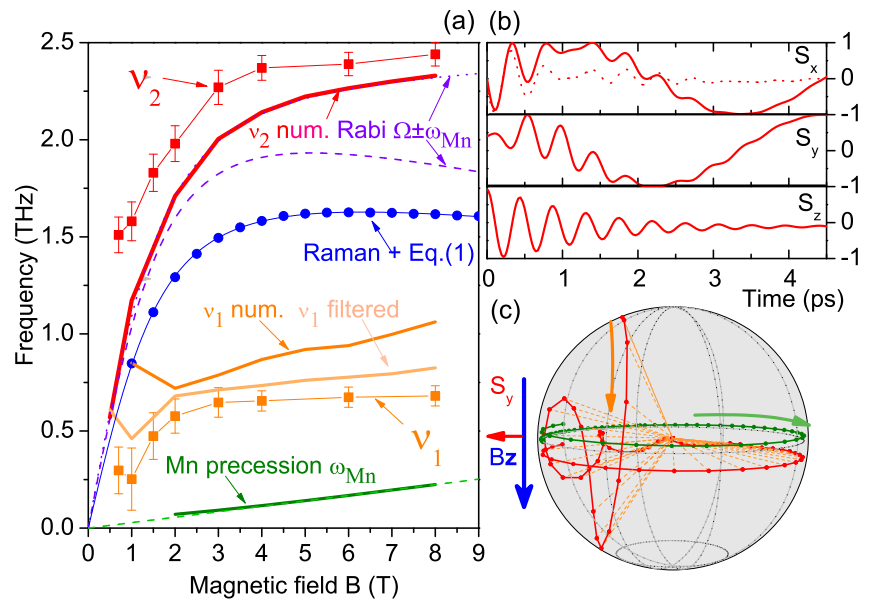

FIG. 3. (Color online). (a) $\nu_{1}$ (orange squares) and $\nu_{2}$ (red squares) are the frequencies present in the transient radiated field. Raman (blue dots) is the optical spin mode frequency determined by Raman measurements. The Raman determination is fitted with Eq.(1) for $x=1.75 \%, T=2.34 \mathrm{~K}$ and $g_{\mathrm{e}}=-1.64$ (blue curve). Rabi are the frequencies given by Eq.(5) (dashed and dotted purple curves). $\omega_{M n}$ are the Mn precession frequencies from theory (dashed green curve) and simulations (solid green curve). $\nu_{1}$ num., $\nu_{2}$ num. and $\nu_{1}$ filtered are frequencies deduced from the simulated $S_{x}(t)$ before (red and orange curves) and after filtering (light orange curve). (b) Normalized $S_{x}(t), S_{y}(t)$ and $S_{z}(t)$ as calculated from Eq.(6) for $B=8 \mathrm{~T}$. The dashed $S_{x}(t)$ curve is obtained after numerical high-pass filtering. (c) $\mathbf{S}(t)$ (red) and $\mathbf{M}(t)$ (green) are the spin trajectories. The external magnetic field $B \mathbf{z}$ is vertical downward. After the pulse, electrons spins have been tilted in the $y$ direction with normalized $S_{y 0}=0.4$, while the Mn spins are parallel to the $y$ axis. The latter precess slowly in the $(x y)$ plane as indicated by the green arrow. Electron spins try to precess around the Mn spins axis as indicated by the orange arrow. After the relaxation of their transverse components (respective to the rotating $\mathrm{Mn}$ precession axis), the electron spins follow the slow Mn precession.

associated to a surface current density carried by each quantum well: $\mathbf{i}_{s}=g_{e} \mu_{B} \mathbf{S}(t) \times \mathbf{y} / w L^{2}$, where $\mathbf{S}(t)=$ $\langle\Psi(t)|\hat{\mathbf{S}}| \Psi(t)\rangle$. Continuity relations of the electromagnetic field at the QW interface leads to the radiated field from each QW after transmission in the air:

$$
\mathbf{e}_{R}(t)=\frac{2 c \mu_{0}}{1+n} \frac{g_{e} \mu_{B}}{w L^{2}} S_{x}(t) \mathbf{z}
$$

where $n$ is the refraction index of the substrate. Therefore, the radiated field is polarized parallel to the $z$-axis, consistent with the experimental observations. Its instantaneous value is proportional to the transverse component $S_{x}(t)$, and its amplitude proportional to the number of spins, thus, to the spin-polarization degree $\zeta$ that saturates with $B$ as the Zeeman energy of Eq.(1) does. As shown in Fig.1(b), the measured amplitude of the radiated field reproduces this behavior. The transverse component of the spin is induced by the optical pulse. Consequently, the radiated amplitude is maximum for a 
pulse energy for which the spins are totally tilted out of their equilibrium axis. Fig.2(b) shows the trend of a saturation of the emitted amplitude when the energy per pulse is further increased from $\simeq 80 \mathrm{~nJ} / \mathrm{cm}^{2}$. Thus, the emitted amplitude naturally departs from the calculations of $\operatorname{Eqs}(3)-(4)$ valid to lowest order in the optical field. A better agreement in the high energy regime has been obtained when going beyond this approximation by resolving the intra-pulse spin dynamics to all orders[18]. A similar non-linear behavior has been found in Ref.[28].

The frequency analysis shown in Fig.2(d) reveals two main frequencies $\nu_{1}$ and $\nu_{2}$. The two frequencies present in the transients are extracted by a linear prediction algorithm[18] and plotted in Fig.3(a). The fitted signal is superposed in Fig.1(a). In Fig.3(a), the two frequencies $\nu_{1}$ and $\nu_{2}$ are compared with the precession frequency of the optical spin mode determined by Raman spectroscopy (blue dots) [14]. The latter matches $Z(B, T) / h$ (blue curve) where $Z$ is given in Eq.(1). However, although both $\nu_{1}$ and $\nu_{2}$ frequencies exhibit a similar magnetic field dependence they are not directly associated with $Z(B, T) / h$ and appear as two frequencies almost symmetrically split from $Z(B, T) / h$.

The transient at $B=8 \mathrm{~T}$ shows that the probed spin component $S_{x}(t)$ experiences quick changes of its phase. This strongly suggests that its precession axis evolves in time. The phenomenon is similar to a magnetic resonance, where a magnetic field $\mathbf{b}_{1}$ oscillates transversally to the polarizing magnetic field $B \mathbf{z}$. Here, the oscillating field is the strong exchange field of the Mn spins which acts as a torque $\alpha \mathbf{S} \times \mathbf{M} . \mathbf{M}(t)$ precesses around the $\mathbf{z}$ axis at $\omega_{M n}=g_{M n} \mu_{B} B / \hbar$ (see lowest green line in Fig.3(a)), since we can neglect the action of the exchange field from the electrons[18]. Due to the transverse oscillation of the Mn-exchange field, the $z$-component of the electron spin $S_{z}(t)$ oscillates at the Rabi pulsation:

$$
\Omega=\frac{1}{\hbar} \sqrt{\left(\alpha M_{y 0}\right)^{2}+\left(\omega_{M n}-\alpha M_{z 0}+\left|g_{e}\right| \mu_{B} B\right)^{2}}
$$

and the transverse component $S_{x}(t)$ has a motion composed of three sinusoids at pulsations $\omega_{M n}$ and $\Omega \pm$ $\omega_{M n}$. The corresponding three frequencies are plotted in Fig.3(a) (green dashed and purple dotted and dashed curves). $\Omega+\omega_{M n}$ matches $\nu_{2}$ within $10 \%$ when setting $M_{z 0}=0$, i.e. when assuming that the Mn spins have been tilted totally out of the plane after the pulse. However, $\nu_{1}$ does not match $\Omega-\omega_{M n}$.

In order to understand the discrepancy concerning $\nu_{1}$, we solved numerically the set of coupled equations of motion for $\mathbf{S}(t)$ and $\mathbf{M}(t)$ (see Ref.[18] for details):

$$
\frac{d \mathbf{S}}{d t}=\left|g_{e}\right| \tilde{\mu}_{B} B \mathbf{S} \times \mathbf{z}+\tilde{\alpha} \mathbf{S} \times \mathbf{M}-\frac{\alpha_{e}}{\left|S_{z i}\right|} \mathbf{S} \times \frac{d \mathbf{S}}{d t}
$$

where $\tilde{\mu}_{B}=\mu_{B} / \hbar$ and $\tilde{\alpha}=\alpha / w L^{2} / \hbar, \alpha_{e}$ is a Gilbert damping parameter. $\mathbf{M}(t)$ is driven by a similar equation[18]. The time evolution of $\mathbf{S}(t)$ and spin trajectories are plotted in Fig.3(b) and Fig.3(c) respectively. To account for the fast electron spin relaxation rate $(3$ ps), $\alpha_{e}$ was set to 0.07 , while for the slower Mn relaxation, we used $\alpha_{M n}=0.01$. From Fig.3(c), we see that the electron spins try to precess around the oscillating $\mathbf{M}(t)$ axis. The damping relaxes its components transverse to the oscillating axis, hence after $3 \mathrm{ps}, \mathbf{S}(t)$ follows $\mathbf{M}(t)$.

A detailed frequency analysis of the simulated $S_{x}(t)$ results in three characteristic frequencies. The highest frequency $\left(\nu_{2}\right.$ num., solid red curve in Fig.3(a)) is in very good agreement with $\Omega+\omega_{M n}$ and hence, $\nu_{2}$; the lowest frequency corresponds to $\omega_{M n}$ and the intermediate one ( $\nu_{1}$ num., solid orange curve) is close to $\nu_{1}$ as shown in Fig.3(a). The $\omega_{M n}$ pulsation is not seen in the transient of Fig.2(a) because the detection process of the $\mathrm{THz}$ field acts as a sharp high pass-filter with cut-off frequency around $0.3 \mathrm{THz}$. To account for this, we have filtered the simulated $S_{x}(t)$ with a numerical high-pass filter[18]. The filtered trajectory of $S_{x}(t)$ is plotted in Fig.3(b). The filtering has a negligible effect on $\nu_{2}$ num., but slightly shifts $\nu_{1}$ num. to lower values $\left(\nu_{1}\right.$ filtered, solid light orange curve), increasing its agreement with $\nu_{1}$. To summarize, the identification of frequencies observed in the transient is supported by the theory within a $10 \%$ quantitative agreement, when taking into consideration the non-linear coupled spin dynamics between the electrons and the Mn ions. The time dependent coupling between these two systems splits the observed frequencies from $Z / h$. This mechanism explains in addition the difference with the Raman determination of the precession modes. Raman probes spin fluctuations, therefore the Mn spins are negligibly disturbed such that $M_{y 0} \simeq 0$, electrons are sensitive to a static exchange magnetic field aligned with the $z$ axis.

In conclusion, we have measured $\mathrm{THz}$ transient fields radiated from optically generated spin-wave excitations in DMS multiple quantum wells. The radiation is polarized parallel to the static magnetic field axis and appears only with a circular polarized pulse. Thus, it's origin cannot be attributed to charge excitations. The spin-wave origin of the emitted field is supported by first-principles theoretical analysis of all aspects of the experiment, including, the generation mechanism, spin dynamics and the properties of emitted radiation. Hence, we provide evidences that DMS quantum wells constitute a highly promising test-bed for deeper investigations in the basic issues of spin-based $\mathrm{THz}$ emission, like for instance, addressing the intrinsic spin precession decay caused by the radiation[29] or manipulating the coupling between spin and radiation at the quantum level, e.g., by inserting such DMS quantum wells into cavities[30, 31].

F.P. thanks L. Thevenard for detailed discussions on GaMnAs. Authors thank M. Wiater for expert technical assistance in the MBE growth and acknowlege the fund- 
ing of CNANO IdF and ANR, the research program of Mairie de Paris and the ERDF European Union grant POIG.01.01.02-00-008/08.

* Corresponding author: florent.perez@insp.jussieu.fr

$\dagger$ Present address: Centro Atómico Bariloche, Bariloche, Argentina.

[1] S. A. Wolf, A. Y. Chtchelkanova \& D. M. Treger, IBM Journal of Research and Development 50, 101 (2006).

[2] J. Kono, Nat Photon 5, 5 (2011).

[3] E. Beaurepaire et al., Applied Physics Letters 84, 3465 (2004).

[4] K. Yamaguchi, M. Nakajima \& T. Suemoto, Phys. Rev. Lett. 105, 237201 (2010).

[5] J. Nishitani, T. Nagashima, and M. Hangyo, Phys. Rev. $B$ 85, 174439 (2012).

[6] T. Kampfrath et al., Nat Photon 5, 31 (2011).

[7] H. Ohno, Science 281, 951 (1998).

[8] J.K. Furdyna, J. Appl. Phys. 64, R29 (1988).

[9] F. Perez, J. Cibert, M. Vladimirova \& D. Scalbert, Phys. Rev. B 83, 075311 (2011).

[10] J. König, H. Lin \& A. H. MacDonald, Phys. Rev. Lett. 84, 5628 (2000).

[11] J.B. Héroux, Y. Ino, M. Kuwata-Gonokami,Y. Hashimoto,S. Katsumoto, Appl. Phys. Lett. 88, 221110-1 (2006)

[12] F. J. Teran et al., Phys. Rev. Lett. 91, 077201 (2003).

[13] B. Jusserand et al., Phys. Rev. Lett. 91, 086802 (2003).

[14] F. Perez et al., Phys. Rev. Lett. 99, 026403 (2007).
[15] C. Aku-Leh, F. Perez, B. Jusserand, D. Richards \& G. Karczewski, Phys. Rev. B 83, 035323 (2011).

[16] M. Vladimirova et al., Phys. Rev. B 78, 081305 (2008).

[17] P. W. Jacobs, F. Perez, C. Aku-leh, R. Merlin \& G. Karzewski, AIP Conference Proceedings 1199, 423 (2010).

[18] See Supplemental Material at [URL...] for details on the sample structure, the $\mathrm{THz}$ setup, the intermediate calculations of equations present in the text.

[19] J. Gaj, R. Planel \& G. Fishman, Solid State Communications 29, 435 (1979).

[20] J. M. Bao, L. N. Pfeiffer, K. W. West \& R. Merlin, Phys. Rev. Lett. 92, 236601 (2004).

[21] D. Popova, A. Bringer, S. Blügel, Phys. Rev. B 84, 214421 (2011).

[22] J. Nishitani, K. Kozuki, T. Nagashima \& M. Hangyo, Applied Physics Letters 96, 221906 (2010).

[23] B. A. Piot et al., Phys. Rev. B 82, 081307 (2010).

[24] C. Weiss, R. Wallenstein \& R. Beigang, Applied Physics Letters 77, 4160 (2000).

[25] A. V. Kimel et al., Nature 435, 655 (2005).

[26] P. S. Pershan, J. P. Van der Ziel \& L. D. Malmstrom, Phys. Rev. 143, 574 (1966).

[27] T. Higuchi, N. Kanda, H. Tamaru \& M. KuwataGonokami, Phys. Rev. Lett. 106, 047401 (2011).

[28] S. G. Carter, Z. Chen \& S. T. Cundiff, Phys. Rev. B 76, 201308 (2007).

[29] M. C. Hickey \& J. S. Moodera, Phys. Rev. Lett. 102, 137601 (2009).

[30] Ö. O. Soykal \& M. E. Flatté, Phys. Rev. Lett. 104, 077202 (2010).

[31] J. Li et al., Opt. Express 19, 22550 (2011). 


\title{
Supplementary Material for : \\ Terahertz radiation from magnetic excitations in diluted magnetic semiconductors
}

\author{
R. Rungsawang, ${ }^{1}$ F. Perez, ${ }^{2, *}$ D. Oustinov, ${ }^{1}$ J. Gómez, ${ }^{2, \dagger}$ V. Kolkovsky ${ }^{3}$ G. \\ Karczewski ${ }^{3}$ T. Wojtowicz, ${ }^{3}$ J. Madéo, ${ }^{1}$ N. Jukam, ${ }^{1}$ S. Dhillon, ${ }^{1}$ and J. Tignon ${ }^{1}$ \\ ${ }^{1}$ Laboratoire Pierre Aigrain, Ecole Normale Supérieure, CNRS (UMR 8551), \\ Université P. et M. Curie, Université D. Diderot, 75231 Paris Cedex 05, France. \\ ${ }^{2}$ Institut des Nanosciences de Paris, CNRS (UMR7588), Université Paris VI, Paris 75005, France \\ ${ }^{3}$ Institute of Physics, Polish Academy of Sciences, 02-668 Warsaw, Poland
}

(Dated: February 28, 2013)

\section{Samples}

The sample is a multi-quantum well (QW) with 20 QWs. The sample has a periodic $\mathrm{Cd}_{1-x} \mathrm{Mn}_{x} \mathrm{Te} / \mathrm{CdMgTe}$ design grown by molecular beam epitaxy on a (100) GaAs substrate. The period includes: a $20 \mathrm{~nm}$ thick $\mathrm{Cd}_{1-x} \mathrm{Mn}_{x}$ Te QW, a 20 $\mathrm{nm}$ thick $\mathrm{Cd}_{0.8} \mathrm{Mg}_{0.2} \mathrm{Te}$ spacer, a $5 \mathrm{~nm}$-thick iodine donors layer and a $50 \mathrm{~nm}$ thick $\mathrm{Cd}_{0.8} \mathrm{Mg}_{0.2}$ Te barrier. 2DEG originates from electrons tunneling from the I doping layer to the QW.

\section{THz measurements}
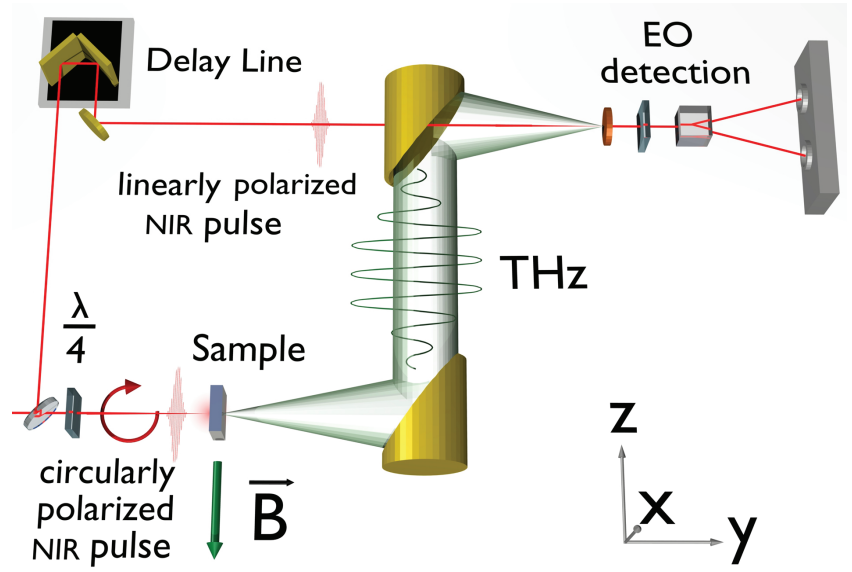

FIG. 1. A near-infrared (NIR) pulse (100 fs) with circular polarization is focused on the QW structure. The sample is immersed in a bath of superfluid helium inside a split-coil magnet with a static field $B$ up to $8 \mathrm{~T}$. The magnetic field is applied along the quantum well plane parallel to the $z$ direction. The optical beam incidence is normal to the QW plane. The $\mathrm{THz}$ radiation is collected by two off-axis parabolic mirrors and detected by free-space electro-optic (EO) sampling.

As depicted on figure.1, the sample was immersed in a bath of superfluid helium with a split-coil superconducting magnet yielding a static field $B$ up to $8 \mathrm{~T}$. The magnetic field was applied along the quantum well plane (Voigt geometry). Near-infrared (NIR) excitation pulses were generated from a mode-locked Ti:sapphire laser at $76 \mathrm{MHz}$ repetition rate. The central wavelength was set to match the 2DEG optical resonance at $763 \mathrm{~nm}$, obtained from photoluminescence measurements. An acousto-optic modulator operated at $8 \mathrm{kHz}$ modulated the laser intensity. The beam was circularly po- larized and focused onto the sample surface with a Gaussian beam waist of $1.6 \mathrm{~mm}$ diameter. The multi-QW layer facing the incoming beam was attached to a sample holder with a $3 \mathrm{~mm}$-diameter aperture. Radiation was collected in transmission geometry. The amplitude and phase the radiation were measured using the phase sensitive detection technique called electro-optic sampling [1]. The electro-optic crystal was a 2 mm-thick (110) ZnTe crystal .

\section{Raman generation mechanism, inverse Faraday effect and radiated field amplitude}

Consider the impulsive electromagnetic field, centered at the wavelength $\lambda_{0}\left(\omega_{0}=2 \pi c / \lambda_{0}\right)$, circularly polarized in the plane $(\mathbf{x}, \mathbf{z})$ of the sample: $\mathbf{E}(t)=$ $\operatorname{Re}\left[(\mathbf{x}+i \mathbf{z}) \sqrt{\frac{E_{P}}{2 \pi c \varepsilon_{0} \omega_{0}}} e^{i \omega_{0} t} \int \tilde{e}_{\omega} e^{i \omega t} d \omega\right]$, where $\tilde{e}_{\omega}$ is the dimensionless normalized Fourier transform of the field $\left(\frac{\lambda_{0}}{2 \pi c} \int|\tilde{e}(\omega)|^{2} d \omega=1\right)$ and $E_{P}$ is the pulse energy per surface unit. The electromagnetic field couples to the crystal through the Hamiltonian: $\hat{H}(t)=-e \mathbf{A}(t) \cdot \hat{\mathbf{p}} / m^{*}$. Before the pulse hits the sample $(t=-\infty)$, the crystal is in its equilibrium state $|i\rangle$. During the pulse action the system is in the state:

$$
|\Psi(t)\rangle=c_{i}(t) e^{-i \omega_{i} t}|i\rangle+\sum_{m} c_{m}(t) e^{-i \omega_{m} t}|m\rangle
$$

where $|m\rangle$ are excited quantum states. We aim at finding $c_{f}(t)$ where $|f\rangle$ is the spin wave state. For that, we write the equation of motion, project it, eliminate the $c_{m}(t)$ for $m \neq i, f$ and find the set of coupled equations:

$$
\begin{gathered}
c_{i}(t)=1-\frac{1}{\hbar^{2}} \sum_{n} \int_{-\infty,-\infty}^{t, t^{\prime}}\left\langle i\left|\hat{H}\left(t^{\prime}\right)\right| n\right\rangle\left\langle n\left|\hat{H}\left(t^{\prime \prime}\right)\right| i\right\rangle c_{i}\left(t^{\prime \prime}\right) \\
\times e^{-i \omega_{n i} t^{\prime}} e^{-i \omega_{i n} t^{\prime \prime}} d t^{\prime} d t^{\prime \prime} \\
-\frac{1}{\hbar^{2}} \sum_{n} \int_{-\infty,-\infty}^{t, t^{\prime}}\left\langle i\left|\hat{H}\left(t^{\prime}\right)\right| n\right\rangle\left\langle n\left|\hat{H}\left(t^{\prime \prime}\right)\right| f\right\rangle c_{f}\left(t^{\prime \prime}\right) \\
\times e^{-i \omega_{n i} t^{\prime}} e^{-i \omega_{f n} t^{\prime \prime}} d t^{\prime} d t^{\prime \prime}
\end{gathered}
$$




$$
\begin{gathered}
c_{f}(t)=-\frac{1}{\hbar^{2}} \sum_{n} \int_{-\infty,-\infty}^{t, t^{\prime}}\left\langle f\left|\hat{H}\left(t^{\prime}\right)\right| n\right\rangle\left\langle n\left|\hat{H}\left(t^{\prime \prime}\right)\right| i\right\rangle c_{i}\left(t^{\prime \prime}\right) \\
-\frac{1}{\hbar^{2}} \sum_{n} \int_{-\infty,-\infty} \iint^{-i \omega_{n f} t^{\prime}} e^{-i \omega_{i n} t^{\prime \prime}} d t^{\prime} d t^{\prime \prime} \\
\left.e^{-i \omega_{n f} t^{\prime}} e^{-i \omega_{f n} t^{\prime \prime}} d t^{\prime} d t^{\prime \prime}\right)|n\rangle\left\langle n\left|\hat{H}\left(t^{\prime \prime}\right)\right| f\right\rangle c_{f}\left(t^{\prime \prime}\right)
\end{gathered}
$$

where $|n\rangle$ are intermediate states involving a hole in the valence band (in contrast to $|i\rangle$ and $|f\rangle$ which have no holes).

Solving these equations yields the dynamics of the state during the pulse and the exact final state after the pulse action. In the manuscript we used a 1st order approximation which consists of solving the system analytically by keeping only terms to lowest order in the field:

$$
\begin{gathered}
c_{i}=1 \\
c_{f}=i \frac{\pi}{\hbar^{2}} \int \sum_{n} \frac{\left\langle f\left|\tilde{H}_{\omega-\omega_{f i}}\right| n\right\rangle\left\langle n\left|\tilde{H}_{\omega}^{*}\right| i\right\rangle}{\omega_{n i}-\omega+i \eta_{n}} d \omega
\end{gathered}
$$

where, $\tilde{H}_{\omega}$ is the Fourier transform of $\hat{H}(t)=\int \tilde{H}_{\omega} e^{i \omega t} d \omega$ and $\eta_{n}$ is the decay rate of the intermediate state $|n\rangle$. The results of this approximation are also compared with a full treatment of (2) which has been solved numerically. However, in the full treatment was achieved by neglecting the spin-conserving terms like $\left\langle f\left|\hat{H}\left(t^{\prime}\right)\right| n\right\rangle\left\langle n\left|\hat{H}\left(t^{\prime \prime}\right)\right| f\right\rangle$.

In the following, we detail the calculation of the radiated field in the 1st order approximation.

An efficient Raman generation of a spin-wave quantum state $|f\rangle$ will occur if: (a) $\omega_{f i} \ll \Delta \omega$, where $\Delta \omega$ is the pulse spectral bandwidth ; (b) the central pulse pulsation is resonant with the intermediate optical transition of the crystal $\omega_{n i} ;$ (c) the decay rate $\eta_{n}$ of the intermediate state is low and (d) the two-photon process $\left\langle f\left|\tilde{H}_{\omega-\omega_{f i}}\right| n\right\rangle\left\langle n\left|\tilde{H}_{\omega}^{*}\right| i\right\rangle$ can transfer spin momentum from the state $|i\rangle$ to the state $|f\rangle$. As we will see below, the later is permitted thanks to the resonance with an intermediate state that contains a spin-orbit mixture. Indeed, upon removing the resonance, all the intermediate states become equivalently weighted in Eq.(3) and the closure relation makes them disappear. The resonance boost the role of a particular intermediate state and thanks to the mixing of spin and orbital degrees of freedom occurring in that state $[2,3]$, the light can exchange spin between $|i\rangle$ and $|f\rangle$. We follow the scheme of Ref.[4] to rewrite the particular $c_{f}$ in the following way:

$$
\begin{array}{r}
c_{f}=i \frac{\pi}{\hbar^{2}}\left(\frac{e}{m^{*}}\right)^{2} \int d \omega \sum_{\alpha, \alpha^{\prime}}\left\langle f\left|a_{\alpha}^{+} a_{\alpha^{\prime}}\right| i\right\rangle \times \\
\sum_{\beta} \frac{\left\langle\alpha\left|\mathbf{A}_{\omega-\omega_{f i}} \cdot \hat{\mathbf{p}}\right| \beta\right\rangle\left\langle\beta\left|\mathbf{A}_{\omega}^{*} \cdot \hat{\mathbf{p}}\right| \alpha^{\prime}\right\rangle}{\omega_{\alpha}-\omega_{\beta}-\omega+i \eta_{\beta}}
\end{array}
$$

where $a_{\alpha}^{+}, a_{\alpha^{\prime}}$ are creation-anhilation operators of conduction electron states $|\alpha\rangle=i|S \mathbf{k} \sigma\rangle .|S\rangle$ is the spherical orbit, $\mathbf{k}$ is the in-plane momentum and $\sigma=\uparrow, \downarrow$ labels the spin. $|\beta\rangle$ is a valence-band state that due to the quantum well confinement is a combination of light and heavy-hole states. The light-hole states in Zinc-Blende semiconductors possess the spin-mixture to obtain the inverse Faraday effect. The latter write: $|\beta\rangle=\alpha_{ \pm 1 / 2}\left|J=\frac{3}{2}, J_{z}= \pm \frac{1}{2}\right\rangle=$ $\alpha_{ \pm 1 / 2}\left[-\sqrt{\frac{2}{3}}|Z \pm \uparrow\rangle \pm \sqrt{\frac{1}{6}}|(X+i Y) \mp \uparrow\rangle\right] . \quad|X\rangle$ is a $\pi$ orbital in the $x$ direction. Thus, a $z$-polarized photon couples an electron $|S \uparrow\rangle$ with $\left|J=\frac{3}{2}, J_{z}=\frac{1}{2}\right\rangle$ and a $x$-polarized photon couples the same state with an electron $|S \downarrow\rangle$. Owing to this selection rule, spin-momentum transfer is forbidden for a linearly polarized pulse, but allowed to a circularly polarized pulse with both polarizations $x$ and $z$ present. In addition, the resonance occurs when $\omega$ is close to the electron-hole pair transition: $\omega_{\alpha}-\omega_{\beta} \simeq \omega_{e-l h}$. Given the approximation that the denominator in Eq.(4) does not depend on $\alpha$ and $\beta$ anymore, and using the form of the electron and hole states, we obtain:

$$
c_{f}=i \int \underbrace{\frac{i 2 \pi}{3 \hbar^{2}} \frac{\left|\frac{e}{m^{*}}\left\langle S\left|\hat{p}_{x}\right| X\right\rangle\right|^{2}}{\omega_{e-l h}-\omega+i \eta_{e-l h}}}_{\chi_{f}(\omega)}\left\langle f\left|\mathbf{A}_{\omega-\omega_{f i}} \times \mathbf{A}_{\omega}^{*} \cdot \hat{\mathbf{S}}\right| i\right\rangle d \omega
$$

where $\left|\left\langle S\left|\hat{p}_{\alpha}\right| \alpha\right\rangle\right|^{2} / m^{*} \simeq 88 \mathrm{eV}$, is the Kane momentum for $\mathrm{CdTe}$, identical for $\alpha=X, Y, Z$. The above form is consistent with the inverse Faraday effect in cubic crystals [5]. Using the form of the circular field given above, we further obtain:

$$
c_{f}=i\left\langle f\left|\hat{S}_{y}\right| i\right\rangle \frac{\lambda_{0} \times e^{2} \times E_{P}}{12 \pi \times \varepsilon_{0} m^{*} c^{2} \times \hbar^{2} \omega_{0}^{2}} \frac{\left|\left\langle S\left|\hat{p}_{x}\right| X\right\rangle\right|^{2}}{m^{*}} I\left(\lambda_{0}\right)
$$

with,

$$
I\left(\lambda_{0}\right)=\int \frac{\tilde{e}_{\omega-\omega_{f i}} \tilde{e}_{\omega}^{*}}{\omega_{e-l h}-\omega_{0}-\omega+i \eta_{e-l h}} d \omega
$$

It makes appear the electron classical radius $e^{2} / \varepsilon_{0} m^{*} c^{2} \simeq$ $0.34 \mathrm{pm}$. We now inject $c_{f}$ into Eq.(1) and keep only linear terms in $c_{f}$ to obtain:

$$
\begin{array}{r}
\left\langle\hat{S}_{x}(t)\right\rangle=n_{2 D} L^{2} \zeta \frac{\lambda_{0} \times e^{2} \times E_{P}}{24 \pi \times \varepsilon_{0} m^{*} c^{2} \times \hbar^{2} \omega_{0}^{2}} \frac{\left|\left\langle S\left|\hat{p}_{x}\right| X\right\rangle\right|^{2}}{m^{*}} \times \\
\left|I\left(\lambda_{0}\right)\right| \cos \left(\omega_{f i} t-\varphi_{0}\right)
\end{array}
$$

we have used $\left\langle f\left|\hat{S}_{y}\right| i\right\rangle\left\langle i\left|\hat{S}_{x}\right| f\right\rangle \simeq-i n_{2 D} L^{2} \zeta / 4$ and $I\left(\lambda_{0}\right)=\left|I\left(\lambda_{0}\right)\right| e^{i \varphi_{0}}$.

From this, using Eq.(4) of the manuscript, we get the amplitude of the radiated field :

$$
\left|\mathbf{e}_{R}\right|=\frac{c \mu_{0} g_{e} \mu_{B}}{1+n} \frac{n_{2 D}|\zeta|}{w} \frac{\lambda_{0} \times 0.34 \mathrm{pm} \times 88 \mathrm{eV} \times \tilde{E}_{P}}{12 \pi \times \hbar^{2} \omega_{0}^{2}}\left|I\left(\lambda_{0}\right)\right|
$$

where $\tilde{E}_{P}$ takes into account the transmission coefficient of the Air/CdMgTe Interface and also the $\alpha_{ \pm 1 / 2}$ factor:

$$
\tilde{E}_{P}=E_{P} \times t_{A i r / C d T e}^{2} \times\left|\alpha_{1 / 2}\right|^{2}
$$




\section{Spin dynamics equations and simulations}

The Hamiltonian of the 2DEG and Mn spins embedded in the $\mathrm{Cd}_{1-x} \mathrm{Mn}_{x}$ Te QW writes:

$$
\begin{gathered}
\hat{H}=\hat{H}_{\text {Kin }}+\hat{H}_{\text {Coulomb }}+\hat{H}_{s-d}+\hat{H}_{\text {Zeeman }} \\
\hat{H}_{s-d}=-\alpha \iiint \hat{\mathbf{S}}(\mathbf{r}) \cdot \hat{\mathbf{M}}(\mathbf{r}) d^{3} r \\
\hat{H}_{\text {Zeeman }}=g_{\mathrm{e}} \mu_{\mathrm{B}} \hat{\mathbf{S}} \cdot \mathbf{B}+g_{\mathrm{Mn}} \mu_{\mathrm{B}} \hat{\mathbf{M}} \cdot \mathbf{B}
\end{gathered}
$$

where $\alpha$ is the exchange coupling between conduction electrons and Mn spins $(\alpha>0)$, and $g_{e}$ and $g_{\mathrm{Mn}}$ are the normal g-factors of, respectively, conduction electrons and Mn electrons. In the convention where $\mu_{\mathrm{B}}>0$, we have $g_{\mathrm{e}} \simeq-1.64$ and $g_{\mathrm{Mn}} \simeq 2.00$. $\mathbf{B}=B \mathbf{z}$ is the external magnetic field applied in the plane of the QW.

We have introduced two vector operators: $\hat{\mathbf{S}}(\mathbf{r})=$ $\chi^{2}(y) \sum_{i} \hat{\mathbf{s}}_{i} \delta\left(\mathbf{r}_{/ /}-\mathbf{r}_{i / /}\right)$ is the 3D electron spin density in a splitted coordinates frame $\mathbf{r}=\left(\mathbf{r}_{/ /}, y\right)$ with $\mathbf{r}_{/ /}$, the inplane position and $y$ the out of plane coordinate. $\chi(y)$ is the electron envelope-function of the first subband of the QW. The $i$ index accounts for the $i$-th electron of the 2DEG, its spin $\frac{1}{2}$ is described by the operator $\hat{\mathbf{s}}_{i}$ and its position is $\mathbf{r}_{i / /} \cdot \hat{\mathbf{M}}(\mathbf{r})=\sum_{j} \hat{\mathbf{I}}_{j} \delta\left(\mathbf{r}-\mathbf{R}_{j}\right)$ is the Mn 3D spin density. The $j$-th $\frac{5}{2}$-spin $\hat{\mathbf{I}}_{j}$ of a single Mn impurities is localized on the cation site $\mathbf{R}_{j} . \hat{\mathbf{S}}=\iiint \hat{\mathbf{S}}(\mathbf{r}) d^{3} r=\sum_{i} \hat{\mathbf{s}}_{i}$ and $\hat{\mathbf{M}}=\iiint \hat{\mathbf{M}}(\mathbf{r}) d^{3} r=\sum_{j} \hat{\mathbf{I}}_{j}$ are the macroscopic spin operators for electrons and $\mathrm{Mn}$ respectively. In the equilibrium state at temperature $T$, each Mn spin assumes the average value $\left\langle\hat{I}_{z}\right\rangle(B, T)$, which is the thermodynamic average over the five occupied states of the Mn atom d-shell, given by the modified Brillouin function[6]. The 2DEG has the equilibrium spin polarization $\zeta=\left(n_{\uparrow}-n_{\downarrow}\right) / n_{2 \mathrm{D}}$.

From the above Hamiltonian, we derive dynamical equations for the electron and Mn macroscopic spin operators, respectively (see Ref.[7] for further details). Then, we take the quantum average spins : $\mathbf{S}(t)=\langle\hat{\mathbf{S}}\rangle$ and $\mathbf{M}(t)=\langle\hat{\mathbf{M}}\rangle$ and make the random phase approximation $\langle\hat{\mathbf{S}} \times \hat{\mathbf{M}}\rangle \simeq$ $\langle\hat{\mathbf{S}}\rangle \times\langle\hat{\mathbf{M}}\rangle$, to obtain the following dynamical equations:

$$
\begin{gathered}
\hbar \frac{d \mathbf{S}}{d t}=\left|g_{e}\right| \mu_{B} B \mathbf{S} \times \mathbf{z}+\hbar \tilde{\alpha} \mathbf{S} \times \mathbf{M}-\frac{\hbar \alpha_{e}}{\left|S_{z i}\right|} \mathbf{S} \times \frac{d \mathbf{S}}{d t} \\
\hbar \frac{d \mathbf{M}}{d t}=-g_{M n} \mu_{B} B \mathbf{M} \times \mathbf{z}-\hbar \tilde{\alpha} \mathbf{S} \times \mathbf{M}-\frac{\hbar \alpha_{M n}}{\left|M_{z i}\right|} \mathbf{M} \times \frac{d \mathbf{M}}{d t}
\end{gathered}
$$

where $\tilde{\alpha}=\alpha / w L^{2} / \hbar$. We have introduced Gilbert damping terms $\left(\alpha_{e}\right.$ and $\left.\alpha_{M n}\right)$ accounting for the relaxation. $S_{z i}<$
0 and $M_{z i}<0$ are the equilibrium macroscopic spins of, respectively, electrons and Mns.

The numerical solution of the above coupled equations was carried with the normalized quantities: $\mathbf{S}=-S_{z i} \tilde{\mathbf{S}}$ and $\mathbf{M}=-M_{z i} \tilde{\mathbf{M}}$. The dynamical equations for these normalized quantities are:

$$
\begin{gathered}
\hbar \frac{d \tilde{\mathbf{S}}}{d t}=\left|g_{e}\right| \mu_{B} B \tilde{\mathbf{S}} \times \mathbf{z}+\Delta \tilde{\mathbf{S}} \times \tilde{\mathbf{M}}-\hbar \alpha_{e} \tilde{\mathbf{S}} \times \frac{d \tilde{\mathbf{S}}}{d t} \\
\hbar \frac{d \tilde{\mathbf{M}}}{d t}=-g_{M n} \mu_{B} B \tilde{\mathbf{M}} \times \mathbf{z}-K \tilde{\mathbf{S}} \times \tilde{\mathbf{M}}-\hbar \alpha_{M n} \tilde{\mathbf{M}} \times \frac{d \tilde{\mathbf{M}}}{d t}
\end{gathered}
$$

Where $\Delta=-\hbar \tilde{\alpha} M_{z i}$ is the Overhauser shift of the electron spins and $K=-\hbar \tilde{\alpha} S_{z i}$ is the Knight shift of Mn spins. The former is typically in the meV range, while the latter is in the $\mu \mathrm{eV}$ range. This justifies the derivation of Eq.(5), where we considered the Mn spins as acting on the electrons as an independent exchange field oscillating at $\omega_{M n}$.

\section{Frequencies analysis}

To analyze the frequencies in the experimental transient, we used a backward linear prediction algorithm detailed in Ref.[8]. This algorithm yields, the frequencies, amplitudes, decay rates and phases of sinusoids present in the transient.

The frequency analysis of the simulated curves presented in Fig.3(b) was also carried with the same algorithm.

To account for the detection filtering, the simulated curves, when indicated, were numerically filtered by a Kaiser highpass filter.

* Corresponding author: florent.perez@insp.jussieu.fr

$\dagger$ Present address: Centro Atómico Bariloche, Bariloche, Argentina.

[1] Q. Wu \& X.-C. Zhang Applied Physics Letters 67, 3523 (1995).

[2] J. M. Bao, L. N. Pfeiffer, K. W. West \& R. Merlin, Phys. Rev. Lett. 92, 236601 (2004).

[3] D. Popova, A. Bringer, S. Blügel, Phys. Rev. B 84, 214421 (2011).

[4] F. A. Blum Phys. Rev. B 1, 1125 (1970).

[5] P. S. Pershan, J. P. Van der Ziel \& L. D. Malmstrom, Phys. Rev. 143, 574 (1966).

[6] J. Gaj, R. Planel \& G. Fishman Solid State Communications 29, 435 (1979).

[7] F. Perez, J. Cibert, M. Vladimirova \& D. Scalbert Phys. Rev. B 83, 075311 (2011).

[8] S. Van Huffel, H. Chen \& C. Decanniere, P. Van Hecke J. of Magn. Reson. 110, 228 (1994). 